\title{
BMJ Open Prognostic nomogram for the severity of acute organophosphate insecticide self- poisoning: a retrospective observational cohort study
}

\author{
Ning Dong, ${ }^{1}$ Shaokun Wang, ${ }^{1}$ Xingliang Li, ${ }^{1}$ Wei Li, ${ }^{1}$ Nan Gao, ${ }^{2}$ Li Pang (D) , \\ Jihong Xing ${ }^{1}$
}

To cite: Dong N, Wang S, Li X, et al. Prognostic nomogram for the severity of acute organophosphate insecticide self-poisoning: a retrospective observational cohort study. BMJ Open 2021;11:e042765. doi:10.1136/ bmjopen-2020-042765

- Prepublication history and additional supplemental material for this paper are available online. To view these files, please visit the journal online (http://dx.doi.org/10.1136/ bmjopen-2020-042765).

Received 14 July 2020 Accepted 12 May 2021
Check for updates

(c) Author(s) (or their employer(s)) 2021. Re-use permitted under CC BY-NC. No commercial re-use. See rights and permissions. Published by BMJ.

${ }^{1}$ Department of Emergency, Jilin University First Hospital, Changchun, Jilin, China

${ }^{2}$ Third Clinical Hospital of Changchun Traditional Chinese Medicine University, Changchun, Jilin, China

Correspondence to

Dr Li Pang; pangli@jlu.edu.cn and

Dr Jihong Xing;

xingjh@jlu.edu.cn

\section{ABSTRACT}

Objective To develop a convenient nomogram

for the bedside evaluation of patients with acute organophosphorus poisoning (AOPP).

Design This was a retrospective study.

Setting Two independent hospitals in northern China, the First Hospital of Jilin University and the Lequn Hospital of the First Hospital of Jilin University.

Participants A total of 1657 consecutive patients admitted for the deliberate oral intake of AOPP within 24 hours from exposure and aged $>18$ years were enrolled between 1 January 2013 and 31 December 2018. The exclusion criteria were: normal range of plasma cholinesterase, exposure to any other type of poisonous drug(s), severe chronic comorbidities including symptomatic heart failure (New York Heart Association III or IV) or any other kidney, liver and pulmonary diseases. Eight hundred and thirty-four patients were included.

Primary outcome measure The existence of severely poisoned cases, defined as patients with any of the following complications: cardiac arrest, respiratory failure requiring ventilator support, hypotension or in-hospital death. Results 440 patients from one hospital were included in the study to develop a nomogram of severe AOPP, whereas 394 patients from the other hospital were used for the validation. Associated risk factors were identified by multivariate logistic regression. The nomogram was validated by the area under the receiver operating characteristic curve (AUC). A nomogram was developed with age, white cells, albumin, cholinesterase, blood pH and lactic acid levels. The AUC was 0.875 (95\% Cl 0.837 to 0.913 ) and $0.855(95 \% \mathrm{Cl} 0.81$ to 0.9$)$ in the derivation and validation cohorts, respectively. The calibration plot for the probability of severe AOPP showed an optimal agreement between the prediction by nomogram and actual observation in both derivation and validation cohorts.

Conclusion A convenient severity evaluation nomogram for patients with AOPP was developed, which could be used by physicians in making clinical decisions and predicting patients' prognosis.

\section{INTRODUCTION}

Acute organophosphorus poisoning (AOPP) is one of the most crucial public health
Strengths and limitations of this study

- The study design for the development of our nomo gram was highly targeted to patients with acute organophosphorus poisoning (AOPP)

- The score of the nomogram is easy to calculate to predict the severity for AOPP.

- We have performed an in-depth validation of our nomogram.

- Some potential confounding factors may not have been controlled due to the retrospective design of this study.

concerns globally, especially prevalent in the agricultural societies of low and middleincome countries. ${ }^{2}$ Patients with severe organophosphorus (OP) poisoning tend to be unstable with a high mortality rate. ${ }^{13}$ A quick assessment of patients' severity and prognosis and the initiation of rapid and suitable therapy are vital. As per current practice, the poisoning severity of patients with AOPP is mainly evaluated based on clinical symptoms ${ }^{4}$ and as of now, no widely accepted bedside evaluation system for detecting the severity of patients with AOPP exists.

A nomogram is a visual scoring system based on multiple regression models. The non-representational messages in multiple regression models are rendered more intuitive and easily applicable to computation by nomogram. Nomograms are extensively used in various clinical settings to predict the probability of an event. As early as 1975, Rumack and Matthew had developed a famous nomogram in acetaminophen poisoning. ${ }^{5}$ Besides, nomograms have been widely used in oncology (eg, tumour metastasis, patients' survival rate, disease recurrence and response to treatment), ${ }^{6-8}$ cardiovascular disease and surgical operations. ${ }^{9} 10$ This further 
encouraged the use of a nomogram in the field of toxicological diseases. ${ }^{1112}$

In this study, we aim to create a nomogram for the bedside evaluation of patients with AOPP using objective data on presentation to the emergency department. The emergency physician could use this simple tool to recognise patients with AOPP due to poor prognosis immediately at presentation.

\section{METHODS}

\section{Patients and study design}

Two independent hospitals, including the First Hospital of Jilin University and the Lequn Hospital of the First Hospital of Jilin University, were selected for this retrospective study. Consecutive patients admitted for AOPP in participant hospitals between 1 January 2013 and 31 December 2018 were included. Inclusion criteria comprised the following: patients admitted for deliberate oral intake of AOPP within 24 hours from exposure and aged $>18$ years. Patients were excluded if they had a normal range of plasma cholinesterase (pChe), were exposed to any other type of poisonous $\operatorname{drug}(\mathrm{s})$, had severe chronic comorbidities including symptomatic heart failure (New York Heart Association III or IV), had chronic kidney disease (CKD) received dialysis treatment before admission and had decompensated liver cirrhosis or chronic obstructive pulmonary disease (COPD). pChe activity was determined by ultraviolet spectrophotometry (enzymatic method, reference interval 4300-11 500 $\mathrm{U} / \mathrm{L})$.

Patients from the hospital with a larger sample size (the primary cohort) were used for nomogram derivation, and those from the other hospital formed the validation cohort. The schematic representation of this study is shown in online supplemental figure S1. This was a retrospective observational cohort study; all data were analysed anonymously and the informed consent of patients was waived.

\section{Patient and public involvement}

Patients were not involved in the design, recruitment or conduct of the study.

\section{Diagnosis and definitions}

\section{Acute organophosphorus poisoning}

A patient with AOPP should be diagnosed by the following factors: medical records documenting an oral intake of OP compounds and a pChe level $<4300 \mathrm{U} / \mathrm{L}$ at admission.

\section{Severe AOPP}

The severity of AOPP was mainly assessed depending on clinical presentations, which is difficult to quantify objectively. Detailed data collected at the presentation could not be obtained owing to the retrospective design of the study. Thus, patients with any of the following were considered to be severely poisoned: cardiac arrest, respiratory failure requiring ventilator support, hypotension or in-hospital death.

\section{Hypotension}

Patients with a blood pressure of $<90 / 60 \mathrm{~mm} \mathrm{Hg}$ and requiring vasopressor administration were considered to be hypotensive.

\section{Ventilation support}

Only patients who experienced respiratory failure, and received endotracheal intubation and invasive ventilator assistance were included. These conditions included both acute respiratory failure due to AOPP and intermediate syndrome.

\section{Management of patients with AOPP}

The treatment of patients with AOPP was not standardised owing to the retrospective design of this study. In brief, the usual protocol of patients with AOPP could be described as follows. Gastric lavage (warm water) was used in patients with AOPP who arrived within 4 hours after organophosphate intake if there were no contradictions. Oral admission of mannitol or magnesium sulfate was used for intestinal cleansing. Intravenous injection and intramuscular atropine were used to reach and maintain patients' atropinisation until pChe rise above 2300 $\mathrm{U} / \mathrm{L}(50 \%$ lower limit of cholinesterase). The usages of oximes were depending on the type of OPs. Haemoperfusion was used for decontamination according to the physician's recommendation, especially in severely poisoned patients. Atropinisation was described as a heart rate of more than 80 beats per minute, the chest is clear and sweating stops.

\section{Data collection}

Patients' records were viewed by two independent physicians. Patients' data within 24 hours after admission from inpatient databases of the participant hospitals were collected as the designed original questionnaire. This questionnaire had variables such as age, gender, medical history (hypertension, diabetes, heart failure, COPD, CKD and liver cirrhosis) and laboratory tests (white cell count, blood haematocrit, platelet count, pChe, blood urea nitrogen (BUN), serum creatinine ( $\mathrm{sCr}$ ), albumin, serum amylase, blood gas analysis (regardless of oxygen therapy, including $\mathrm{pH}$, partial pressure of oxygen, partial pressure of carbon dioxide, base excess (BE), lactic acid)). In-hospital complications were also recorded, including ventilator assistance, hypotension, cardiac arrest or death. The outcome of the present study was the presence of severe AOPP (see the Diagnosis and definitions section).

\section{Statistical methods}

Continuous variables were presented as means (SD) or medians (IQR). Student's t-test or Mann-Whitney U test was used for comparison as per their suitability. Categorical variables were presented as numbers (with percentage values) and compared with $\chi^{2}$ or Fisher's exact test. Missing variables (online supplemental table S1) were 
Table 1 Characteristics of patients with AOPP in derivation and validation cohorts

\begin{tabular}{|c|c|c|c|}
\hline Variables & Derivation cohort & Validation cohort & $P$ value \\
\hline Patients (n) & 440 & 394 & - \\
\hline Age (years; median; IQR) & $43.00(33.00-54.25)$ & $45.00(33.00-58.00)$ & 0.274 \\
\hline \multicolumn{4}{|l|}{ Medical history, n (\%) } \\
\hline Hypertension & $30(6.8)$ & $34(8.6)$ & 0.395 \\
\hline \multicolumn{4}{|c|}{ Laboratory tests (median; IQR) } \\
\hline White cells $\left(\times 10^{9} / \mathrm{L}\right)$ & $13.5(8.99-18.08)$ & $13.84(9.79-18.78)$ & 0.172 \\
\hline Haemoglobin (g/L) & $138.5(124-52)$ & $139(123-153)$ & 0.945 \\
\hline Platelets $\left(\times 10^{9} / \mathrm{L}\right)$ & $214(177-259)$ & $215(176-252)$ & 0.78 \\
\hline Albumin ( $g / L)$ & $39.5(35.27-43.00)$ & $39.30(35.2-42.9)$ & 0.646 \\
\hline $\mathrm{sCr}(\mathrm{mg} / \mathrm{dL})$ & $0.67(0.56-0.84)$ & $0.68(0.55-0.86)$ & 0.685 \\
\hline Serum amylase (U/L) & $113(66-261)$ & $91(58-220.7)$ & 0.03 \\
\hline \multicolumn{4}{|c|}{ Blood gas analysis (median; IQR) } \\
\hline $\mathrm{pH}$ & $7.38(7.31-7.43)$ & $7.37(7.29-7.42)$ & 0.258 \\
\hline $\mathrm{PCO}_{2}(\mathrm{~mm} \mathrm{Hg})$ & $37(33-41)$ & $35(31.2-43)$ & 0.636 \\
\hline $\mathrm{PO}_{2}(\mathrm{~mm} \mathrm{Hg})$ & $92(73-122.2)$ & $89(70-120.7)$ & 0.797 \\
\hline Base excess (mmol/L) & $-3.20(-8$ to 0.8$)$ & $-2.75(-6.77$ to 1.28$)$ & 0.121 \\
\hline Lactic acid (mmol/L) & $1.40(0.90-2.9)$ & $1.60(1.00-3.5)$ & 0.004 \\
\hline Severely poisoned, n (\%) & $104(23.6)$ & $97(24.6)$ & 0.802 \\
\hline
\end{tabular}

AOPP, acute organophosphorus poisoning; BUN, blood urea nitrogen; pChe, plasma cholinesterase; $\mathrm{PCO}_{2}$, partial pressure of carbon dioxide; $\mathrm{PO}_{2}$, partial pressure of oxygen; sCr, serum creatinine.

imputed 50 times by chained equations. Only continuous variables such as age, white cell count, haemoglobin, platelets, albumin, pChe, BUN, sCr, serum amylase and blood gas analysis outcomes were used in the imputation model.

Statistical analyses were conducted based on logistic regression to identify risk factors. Variables with a $\mathrm{p}$ value $<0.2$ in univariate logistic regression were considered to be linked to the study outcomes and were further used for backward step-down logistic regression. The regression model with the minimum Akaike's information criterion (AIC) was used in nomogram formulation. Multicollinearity was assessed with correlation matrix and variance inflation factors (VIF, online supplemental figure S2 and table S2). The nomogram's performance was measured by area under the receiver operating characteristic curve (AUC) and assessed by comparing nomogram-predicted versus observed incidences of the outcomes. External validation of the nomogram was conducted by calculating the total points of each patient in the validation cohort as per the established nomogram, followed by logistic regression in this cohort carried out by using the total points as a factor, and last, the AUC and calibration curve was derived based on regression analysis. Two-tailed $\mathrm{p}<0.05$ was considered statistically significant. All the statistical analyses were conducted in R software V.3.4.0 (R Foundation for Statistical Computing, Vienna, Austria). R packages, namely 'mice', '13 'rms', 14 'pROC', 'Hmisc' 16 and 'corrplot', ${ }^{17}$ were used in the analysis.

\section{RESULTS}

\section{Clinical characteristics of patients}

To build the primary cohort, 809 patients with AOPP were found in the electronic database of the First Hospital of Jilin University. Of those, 369 (45.6\%) patients were excluded based on the exclusion criteria, among whom $16(11.5 \%)$ were excluded owing to accidental or skin exposure, $132(35.7 \%)$ for being admitted after 24 hours postexposure, $193(52.3 \%)$ were poisoned in combination 


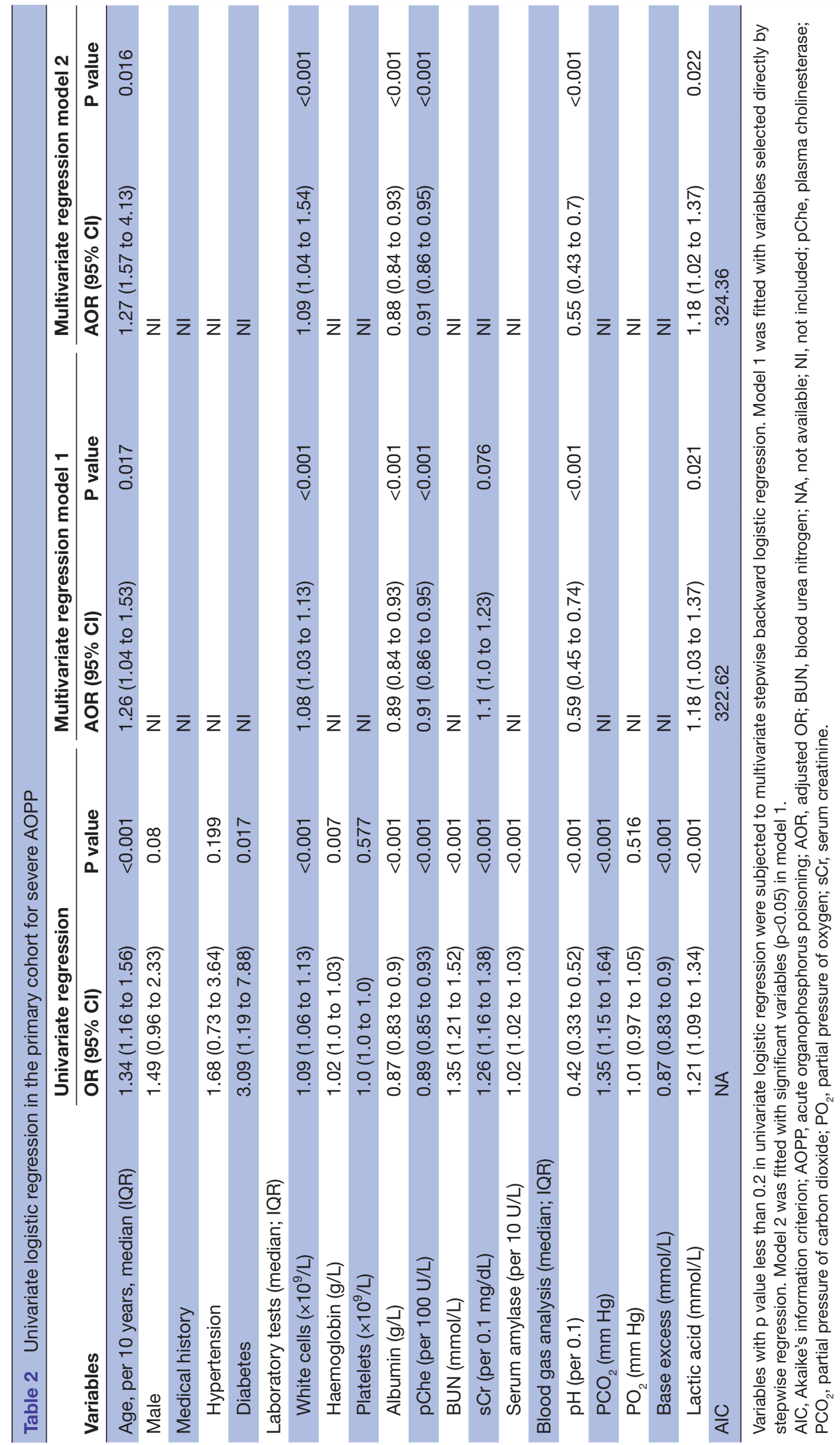

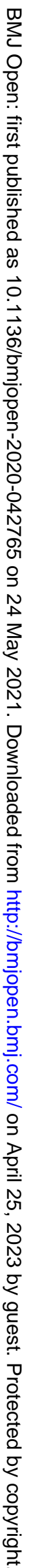




\begin{tabular}{|c|c|c|c|c|c|c|c|c|c|c|c|c|c|c|c|}
\hline Points & 0 & & & 20 & & 30 & 40 & & 50 & 60 & 70 & 80 & 90 & & 100 \\
\hline \multirow{2}{*}{ Age } & & & & & & & & & & & & & & & \\
\hline & 10 & 30 & & 50 & 70 & & 90 & & & & & & & & \\
\hline WBC & 0 & 5 & & 10 & 15 & 20 & 25 & 30 & 35 & 40 & 45 & & & & \\
\hline Albumin & 55 & & 50 & & 45 & & 40 & 35 & 5 & 30 & 25 & 20 & & 15 & \\
\hline PChe & 4500 & 400 & & 3500 & 300 & & 2500 & 2000 & 1500 & 1000 & 500 & 0 & & & \\
\hline PH & 7.7 & & 6 & & 7.5 & 7.4 & & 7.3 & 7.2 & 7. & & 7 & 6.9 & & 6.8 \\
\hline Lactic acid & 0 & 2 & 4 & 6 & 8 & 10 & 12 & 14 & 16 & & & & & & \\
\hline Total Points & 0 & & & 50 & & 100 & & & 150 & 20 & & 250 & & & 300 \\
\hline Risk of severe poisoned & & & & & & & & & & $\begin{array}{l}0.2 \\
0.1 \quad 0\end{array}$ & \begin{tabular}{c|c}
$0.4 \quad 0.6$ \\
3 & 0.5
\end{tabular} & $\frac{0.8}{0.7}$ & .9 & & \\
\hline
\end{tabular}

Figure 1 Prognostic nomogram for severe acute organophosphorus poisoning (AOPP). pChe, plasma cholinesterase; WBC, white blood cell.

with other drugs and 28 (7.6\%) for having normal pChe levels at admission (online supplemental figure S1). Therefore, the derivation cohort included 440 patients. Likewise, the validation cohort was composed of 394 patients from the Lequn Hospital of the First Hospital of Jilin University after excluding 354 patients who did not meet the inclusion criteria (21 $(5.9 \%)$ for accidental or skin exposure, $146(41.2 \%)$ for being admitted after 24 hours postexposure, $169(47.7 \%)$ for being poisoned in

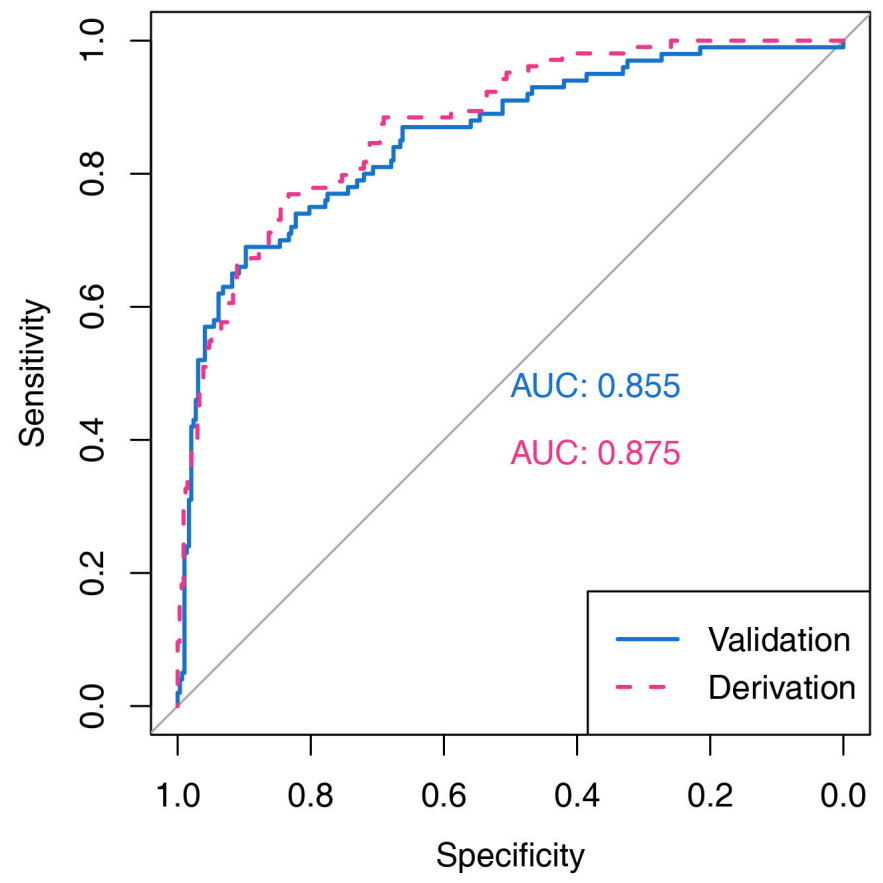

Figure 2 Receiver operating characteristic (ROC) of nomogram in predicting severe acute organophosphorus poisoning (AOPP) in derivation and validation cohorts. AUC, area under the receiver operating characteristic curve. combination with other drugs and $39(11 \%)$ with normal pChe). The flow chart of the patients' recruitment has been given in online supplemental figure S1. The characteristics of patients with AOPP from both groups are provided in table 1 . As per the definition of severe AOPP, the incidence of severely poisoned patients was $23.6 \%$ in the derivation cohort (table 1).

\section{Independent prognostic factors in the primary cohort}

Univariate logistic regression found that factors such as age $(\mathrm{OR}=1.34,95 \%$ CI 1.16 to 1.56$)$, history of diabetes $(\mathrm{OR}=3.09,95 \%$ CI 1.19 to 7.88$)$, white cell count (OR=1.09, 95\% CI 1.06 to 1.13), haemoglobin (OR=1.02, 95\% CI 1.0 to 1.03$)$, albumin ( $\mathrm{OR}=0.87,95 \%$ CI 0.83 to 0.9 ), pChe ( $\mathrm{OR}=0.89,95 \%$ CI 0.85 to 0.93 ), BUN (OR=1.35, 95\% CI 1.21 to 1.52$)$, $\mathrm{sCr}(\mathrm{OR}=1.26$, 95\% CI 1.16 to 1.38$)$, serum amylase ( $\mathrm{OR}=1.02,95 \%$ CI 1.02 to 1.03$)$, blood $\mathrm{pH}(\mathrm{OR}=0.42,95 \%$ CI 0.33 to $0.52), \mathrm{BE}(\mathrm{OR}=0.87,95 \%$ CI 0.83 to 0.9$)$ and lactic acid $(\mathrm{OR}=1.21,95 \%$ CI 1.09 to 1.34$)$ levels were significantly linked to the severity of AOPP. Multivariate analyses established that age (adjusted OR $(\mathrm{AOR})=1.26,95 \%$ CI 1.04 to 1.53 ), white cells (AOR $=1.08,95 \%$ CI 1.03 to 1.13 ), albumin (AOR $=0.89,95 \%$ CI 0.84 to 0.93 ), pChe (AOR $=0.91,95 \%$ CI 0.86 to 0.95$)$, $\mathrm{sCr}(\mathrm{AOR}=1.1,95 \%$ CI 1.0 to 1.23$), \mathrm{pH}(\mathrm{AOR}=0.59,95 \%$ CI 0.45 to 0.74$)$ and lactic acid (AOR=1.18, 95\% CI 1.03 to 1.37 ) levels were independent risk factors for severe AOPP (table 2, multivariate regression model 1 ). The factor sCr was eventually excluded from the final model (table 2, multivariate regression model 2$)$, as its $p$ value $(p=0.076)$ was insignificant, although it could improve the AIC from 324.36 to 322.62. A correlation matrix of enrolled variables and VIF of model 2 showed no multicollinearities among variables (online supplemental figure S1 and table S2). The 
A

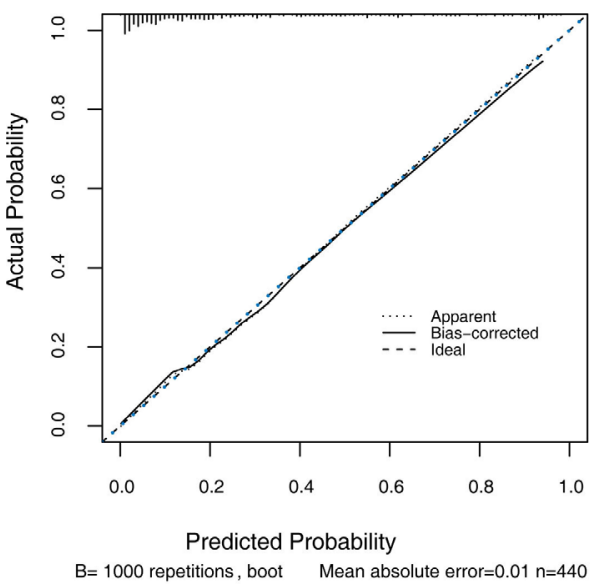

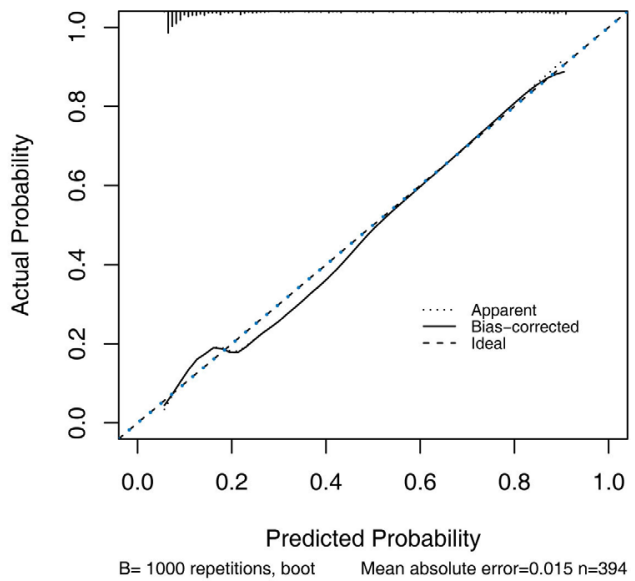

Figure 3 Calibration curve for predicting patients with severe acute organophosphorus poisoning (AOPP) $f$ in the validation (A) and validation cohorts (B); nomogram-predicted probabilities of severity are plotted on the $x$-axis; actual overall survival is plotted on the $y$-axis. The long dashed line (ideal) represents an ideal nomogram whose predicted outcome perfectly corresponds to the actual outcome. The solid line (bias corrected) represents the bootstrap-corrected performance of our nomogram, and the short dashed line (apparent) represents the apparent accuracy of the nomogram. The apparent and biascorrected lines fell approximately along the ideal line, which indicates that the score calculated by the nomogram accurately represents the actual prediction of severe AOPP in both the primary and validation cohorts.

nomogram for severity of AOPP was developed in accordance with the multivariate regression model 2.

\section{Risk prediction nomogram for AOPP severity}

The nomogram was composed of all six significant independent factors for severe AOPP in the derivation cohort (age, white cells, albumin, pChe, blood $\mathrm{pH}$ and lactic acid (figure 1)). With the help of the nomogram, each patient obtained a score indicating a specific probability of developing severe AOPP. Receiver operating characteristic assessed the nomogram's discriminative power, and the AUC for predicting the severity of AOPP was 0.875 (95\% CI 0.837 to 0.913 ; figure 2 ). The calibration plot for the probability of AOPP severity indicated an optimal agreement between the predictions through the nomogram and actual observations (figure $3 \mathrm{~A}$ ). The scores of the nomogram and represented risks are listed in online supplemental table S2.

\section{Validation of predictive accuracy of the nomogram for AOPP severity}

The incidence of severely poisoned cases in the validation cohort was $24.6 \%$ (table 1 ). The nomogram indicated good discriminative ability with an AUC value of 0.855 (95\% CI 0.81 to 0.9 ) (figure 2 ). The calibration curve indicated a good agreement between prediction and observation in the probability of severe AOPP (figure 3B). No significant difference in AUC values between derivation and validation cohorts $(\mathrm{p}=0.497)$ was found.

\section{DISCUSSION}

Eligible patients were patients with AOPP identified from the inpatient database from participating hospitals. Based on the clinical characteristics and laboratory results, we first established and validated prognostic nomograms for severity of patients with AOPP. The proposed nomograms revealed excellent discrimination in both the derivation and validation cohorts. Additionally, accurate predictions for AOPP severity using the proposed nomograms were indicated by calibration curves.

In this study, multivariate logistic regression was used for predicting a binary outcome of developing or not developing severe AOPP. This nomogram was developed based on six predictors, comprising age, white cells, pChe, albumin, blood $\mathrm{pH}$ and lactic acid levels. These variables were established to be linked with a poor prognosis of patients with AOPP in certain earlier studies. ${ }^{18-21}$ Multivariate analysis showed that age had a statistically significant association with severe AOPP. One possible reason for this may be that older patients are more likely to be impaired by OPs due to a weak cholinergic system. ${ }^{22}$ White cell count is a predictor for many poisoning diseases as well as AOPP. ${ }^{23}$ Blood $\mathrm{pH}^{24}{ }^{25}$ and lactic acid level ${ }^{20}$ were associated with a poor prognosis of AOPP in previous studies. Albumin could form serum albumin adducts in the presence of OP compounds. ${ }^{26}{ }^{27}$ Thus, it is reasonable to find a negative association between albumin and severe AOPP. We used butyryl-cholinesterase levels in this study because blood acetylcholinesterase was not available in our hospital and in many other medical centres. Only using butyryl-cholinesterase may overestimate the risk of severe AOPP because some OPs may inhibit butyryl-cholinesterase more effectively than acetylcholinesterase. ${ }^{1}$ The combination of these six indicators in our nomogram may overcome this disadvantage. These variables were objective, easy to acquire and quickly assessable. The variables used in this nomogram may be non-specific and may significantly differ in other diseases, such as acetaminophen poisoning. ${ }^{28}$ Nevertheless, only common laboratory tests may be available in rural areas, 
and more common indicators enlarge the practicality of the nomogram. By combining these non-specific variables, good discrimination in both cohorts was obtained with this nomogram.

The severity of AOPP is related to the severity of inhibition of the cholinesterase system, which may decide by types of OP compounds, doses of intake and time from onset to treatment. Previous studies have discovered that body mass index ${ }^{29}$ and alcohol coingestion ${ }^{30}$ were associated with patients' outcomes. However, due to the retrospective design, these indicators were inaccessible in this study.

The outcome of our study was severe AOPP (patients with any of the following: cardiac arrest, respiratory failure requiring ventilator support, hypotension or in-hospital death) other than mortality. Previous studies have also found a Glasgow Coma Scale (GCS) score $\leq 13$ has good discrimination in predicting mortality of patients with AOPP (AUC 0.84 , sensitivity 0.79 , specificity 0.79 ), Poison Severity Score (PSS) was similarly effective as GCS at predicting such outcome. ${ }^{31}$ However, the GCS and PSS were not available due to the retrospective design of our study. The different definitions of predicting outcome also prevented the direct comparison of the pre-existed scoring system and our nomogram.

We calculated predicted risk and relative scores in the nomogram in online supplemental table S3. As per the nomogram, patients with a $>218$ points (online supplemental table S3) score would have a $>50 \%$ chance of developing severe symptoms of poisoning, a patient with a score of $>245$ or $>260$ would develop a severe case with a probability of $80 \%$ or $90 \%$, respectively. A higher score indicates the need for intensive care, ventilation and blood pressure management, or such cases may turn fatal. Low-risk patients do not need to be admitted to the intensive care unit or even require haemoperfusion. These hypotheses require further prospective investigation. A nomogram is beneficial to emergency medicine physicians for evaluating patients immediately, especially in rural areas. This predictive nomogram may be used in optimally estimating individualised disease-related risks that simplify patient management-related decisionmaking. This user-friendly instrument could be applied by physicians to enhance patient management and to eventually reduce mortality.

This study established a nomogram to be used as a support tool for predicting severe AOPP cases. Nonetheless, certain limitations of this study need mentioning. First, due to this study's retrospective design, some valuable variables such as symptoms and certain clinical risk scores (GCS, Peradeniya Organophosphorus Poisoning Scale, etc) were inaccessible. Thus, the discrimination of this nomogram and other risk scoring systems was not compared. Second, this study enrolled only two independent hospitals. The two cohorts had a significant difference in blood amylase and lactic acid levels; thus, other validation cohorts would encourage widespread use of this nomogram.

\section{CONCLUSION}

A six-variable risk prediction nomogram was developed based on demographic and routine laboratory tests, which accurately predicts the probability of severe AOPP. This nomogram can be used to help risk stratify patients with AOPP on presentation to the emergency department.

Contributors ND: conceptualisation, data curation, formal analysis, investigation, methodology, writing —original draft, writing—review and editing. SW: data curation, formal analysis, validation, visualisation, writing —original draft. XL: data curation, formal analysis, investigation, validation, visualisation, writing—original draft. WL, NG: data collection, investigation, validation, visualisation, writingreview and editing. LP, JX: conceptualisation, formal analysis, investigation, project administration, resources, supervision, validation, writing — review and editing.

Funding The authors have not declared a specific grant for this research from any funding agency in the public, commercial or not-for-profit sectors.

Competing interests None declared.

Patient consent for publication Not required.

Ethics approval This study was approved by the institutional ethics committee of the First Hospital of Jilin University (approval number 2020-594).

Provenance and peer review Not commissioned; externally peer reviewed. Data availability statement Data are available upon reasonable request.

Supplemental material This content has been supplied by the author(s). It has not been vetted by BMJ Publishing Group Limited (BMJ) and may not have been peer-reviewed. Any opinions or recommendations discussed are solely those of the author(s) and are not endorsed by BMJ. BMJ disclaims all liability and responsibility arising from any reliance placed on the content. Where the content includes any translated material, BMJ does not warrant the accuracy and reliability of the translations (including but not limited to local regulations, clinical guidelines, terminology, drug names and drug dosages), and is not responsible for any error and/or omissions arising from translation and adaptation or otherwise.

Open access This is an open access article distributed in accordance with the Creative Commons Attribution Non Commercial (CC BY-NC 4.0) license, which permits others to distribute, remix, adapt, build upon this work non-commercially, and license their derivative works on different terms, provided the original work is properly cited, appropriate credit is given, any changes made indicated, and the use is non-commercial. See: http://creativecommons.org/licenses/by-nc/4.0/.

ORCID iD

Li Pang http://orcid.org/0000-0003-0561-4100

\section{REFERENCES}

1 Eddleston M, Buckley NA, Eyer P, et al. Management of acute organophosphorus pesticide poisoning. Lancet 2008;371:597-607.

2 Yu S-Y, Gao Y-X, Walline J, et al. Role of penehyclidine in acute organophosphorus pesticide poisoning. World J Emerg Med 2020;11:37-47.

3 Hulse EJ, Haslam JD, Emmett SR, et al. Organophosphorus nerve agent poisoning: managing the poisoned patient. $\mathrm{Br} J$ Anaesth 2019;123:457-63.

4 Lin GW, Wang JY, JB G. Organophosphate pesticide poisoning. Pract Int Med 2017:731-2.

5 Rumack $\mathrm{BH}$, Matthew $\mathrm{H}$. Acetaminophen poisoning and toxicity. Pediatrics 1975;55:871-6.

$6 \mathrm{Kim}$ SM, Min B-H, Ahn JH, et al. Nomogram to predict lymph node metastasis in patients with early gastric cancer: a useful clinical tool to reduce gastrectomy after endoscopic resection. Endoscopy 2020;52:435-43.

7 Chen J, Fang A, Chen M, et al. A novel inflammation-based nomogram system to predict survival of patients with hepatocellular carcinoma. Cancer Med 2018;7:5027-35.

8 Zhang $\mathrm{F}$, Huang $\mathrm{M}$, Zhou $\mathrm{H}$, et al. A nomogram to predict the pathologic complete response of neoadjuvant chemotherapy in triple-negative breast cancer based on simple laboratory indicators. Ann Surg Oncol 2019;26:3912-9.

9 Wu J, Quu J, Jiang W, et al. Development and validation of a nomogram predicting the probability of type a aortic dissection at 
a diameter below 55 mm: A retrospective cohort study. Int J Surg 2018;60:266-72.

10 Bedi M, Ethun CG, Charlson J, et al. Is a nomogram able to predict postoperative wound complications in localized soft-tissue sarcomas of the extremity? Clin Orthop Relat Res 2020;478:550-9.

11 Bateman DN. Paracetamol poisoning: beyond the nomogram. $\mathrm{Br} \mathrm{J}$ Clin Pharmacol 2015;80:45-50.

12 Senarathna L, Eddleston M, Wilks MF, et al. Prediction of outcome after paraquat poisoning by measurement of the plasma paraquat concentration. QJM 2009;102:251-9.

13 Groothuis-Oudshoorn K, Buuren SV. \{mice\}: Multivariate Imputation by Chained Equations in R. J Stat Software 2011;45:1-67.

14 Frank E, Harrell J. rms: regression modeling strategies. $R$ package version 6.1-0, 2020. Available: https://CRAN.R-project.org/package= rms

15 Robin X, Turck N, Hainard A, et al. pROC: an open-source package for $\mathrm{R}$ and $\mathrm{S}+$ to analyze and compare $\mathrm{ROC}$ curves. BMC Bioinformatics 2011;12:77.

16 Frank E, Harrell J. Hmisc: Harrell miscellaneous. R package version 4.4-2, 2020. Available: https://CRAN.R-project.org/package=Hmisc

17 Wei T, Simko V. R package "corrplot": visualization of a correlation matrix (Version 0.84), 2017. Available: https://github.com/taiyun/ corrplot

18 Kumar S, Agrawal S, Raisinghani N, et al. Leukocyte count: a reliable marker for the severity of organophosphate intoxication? J Lab Physicians 2018;10:185-8.

19 Tang Y, Hu L, Hong G, et al. Diagnostic value of complete blood count in paraquat and organophosphorus poisoning patients. Toxicol Ind Health 2018;34:439-47.

20 Wu X, Xie W, Cheng Y, et al. Severity and prognosis of acute organophosphorus pesticide poisoning are indicated by $\mathrm{C}$-reactive protein and copeptin levels and APACHE II score. Exp Ther Med 2016;11:806-10.
21 Tang W, Ruan F, Chen Q, et al. Independent prognostic factors for acute organophosphorus pesticide poisoning. Respir Care 2016;61:965-70.

22 Schliebs R, Arendt T. The cholinergic system in aging and neuronal degeneration. Behav Brain Res 2011;221:555-63.

23 Kumar S, Agrawal S, Raisinghani N, et al. Leukocyte count: a reliable marker for the severity of organophosphate intoxication? J Lab Physicians 2018;10:185-8.

24 Gündüz E, Dursun R, Icer $\mathrm{M}$, et al. Factors affecting mortality in patients with organophosphate poisoning. J Pak Med Assoc 2015;65:967-72.

25 Tang W, Ruan F, Chen Q, et al. Independent prognostic factors for acute organophosphorus pesticide poisoning. Respir Care 2016;61:965-70.

26 Tarhoni MH, Lister T, Ray DE, et al. Albumin binding as a potential biomarker of exposure to moderately low levels of organophosphorus pesticides. Biomarkers 2008;13:343-63.

27 Marsillach J, Costa LG, Furlong CE. Protein adducts as biomarkers of exposure to organophosphorus compounds. Toxicology 2013;307:46-54.

28 Ghannoum M, Kazim S, Grunbaum AM, et al. Massive acetaminophen overdose: effect of hemodialysis on acetaminophen and acetylcysteine kinetics. Clin Toxicol 2016;54:519-22.

29 Lee DH, Jung KY, Choi YH, et al. Body mass index as a prognostic factor in organophosphate-poisoned patients. Am J Emerg Med 2014;32:693-6.

30 Eddleston M, Gunnell D, von Meyer L, et al. Relationship between blood alcohol concentration on admission and outcome in dimethoate organophosphorus self-poisoning. Br J Clin Pharmacol 2009;68:916-9.

31 Davies JOJ, Eddleston M, Buckley NA. Predicting outcome in acute organophosphorus poisoning with a poison severity score or the Glasgow coma scale. QJM 2008;101:371-9. 